\title{
A NOTE ON THE THEORY OF SUMMABLE INTEGRALS.
}

BY ML. S. CHAPMAN.

(Read before the American Mathematical Society, December 28, 1910.)

\section{§1. Introduction.}

OF recent years increasing use has been made of infinite series which do not converge; but whereas the mathematicians before the time of Abel and Cauchy used such series without proper examination of the validity of their use, modern mathematicians in general employ them only when the legitimacy of the work can be clearly demonstrated. The need so arising for theories to justify the application of ordinary methods and transformations to non-convergent series just as if they did converge has been fully recognized, and to meet it there have been published many well-known memoirs by Cesàro, Borel, Poincaré, LeRoy, and others. The theory is still being rapidly extended, and new important applications are constantly appearing. Among recent recent workers on the subject may be mentioned Bohr, Bromwich, Fejer, Hardy, C. N. Moore, and Riesz.

Parallel with the theory for infinite series there is a theory for infinite integrals, but the latter has not yet been developed so much as the former. Mr. Hardy* seems to have been the first to define a "summable" integral, and his paper was closely followed by one due to Dr. C. N. Moore, $\dagger$ in which some properties of summable integrals were proved. Subsequently Dr. Bromwich $\ddagger$ wrote on the same subject, and more recently still the theory has been generalized by Mr. Hardy§ and

\footnotetext{
* Quar. Jour. of Mathematics, vol. 35, p. 54.

$\dagger$ "On the introduction of convergence factors into summable series and summable integrals," Trans. Amer. Math. Society, vol. 8, p. 299.

$\ddagger$ "On the limits of certain infinite series and integrals," Math. Annalen, vol. 65 , p. 350 .

\& G. H. Hardy, "Notes on some points in the integral calculus," Messenger of Mathematics, vol. 40. "Theorems connected with Maclaurin's test for the convergence of series," Proc. Lond. Math. Soc. G. H. Hardy and S. Chapman, "A general view of the theory of summability of series and integrals," Quar. Jour. of Mathematics, 1911.
} 
myself.* In particular I have applied methods of "summation" to series and integrals which converge, with the object, not, of course, of finding their sum, but of gaining an insight into the nature of their convergence.

\section{§ 2. Summable Integrals.}

Mr. Hardy's original definition of the summability of an integral was quite analogous to Cesàro's definition of the summability of a series by a first mean. Thus, if $f(x)$ is a function which is integrable in every finite interval lying in the interval $a \leqq x$, then the integral

$$
\int_{a}^{\infty} f(x) d x
$$

is said to be summable $\left(C_{1}\right) \dagger$ if the limit

$$
\lim _{x \rightarrow \infty} \frac{1}{x} \int_{a}^{x} \int_{a}^{a} f(\beta) d \beta d \alpha
$$

exists; and the value of the limit is called the "sum" or "value" of the integral.

The extension of this definition is obvious, viz.,

The integral $\int_{a}^{\infty} f(x) d x$ is summable $\left(C_{r}\right)$ if the limit

$$
\lim _{x \rightarrow \infty} \frac{r !}{x^{r}} \int_{a}^{x} \int_{a}^{a_{1}} \int_{a}^{a_{2}} \cdots \int_{a}^{a_{r}} f(\theta) d \theta d \alpha_{r} d \alpha_{r-1} \cdots d \alpha_{1}
$$

exists; and as before the value of the limit defines the "sum" or "value" of the integral.

For many purposes, however, in the development of the general theory, the above multiple integral is more convenient when it is transformed into the expression

$$
\int_{a}^{x}\left(1-\frac{y}{x}\right)^{r} f(y) d y
$$

which is easily deduced by the theory of multiple integrals.

* S. Chapman, "On non-integral orders of summability of series and integrals," Proc. Lond. Math. Soc., 1911.

†The convenient notation of which this is a particular case was introduced by Mr. Hardy in a paper published in the Proc. Lond. Math. Soc., series 2 , vol. 4 , p. 257. 
In particular the latter form is valuable because it shows the analogy of the above method of summation with that introduced (for the case of series) by Dr. M. Riesz.*

Further, it suggests the idea of non-integral orders of summability, and is an eminently suitable form for proving theorems on summability in full generality and with extreme simplicity.

Dr. C. N. Moore, in the paper already quoted, proved that if an integral is convergent, it is summable $\left(C_{1}\right)$ and its "sum" is the same as the ordinary value. This is a particular case of the more general theorem which I have established, $\dagger$ to the effect that "if an integral is summable $\left(C_{r}\right)$, then it is summable $\left(C_{r^{\prime}}\right)$, with the same sum, provided $r^{\prime}>r$, where $r$ and $r^{\prime}$ may be any positive or negative numbers (including zero), greater than - 1." This may be called the general condition of consistency.

In this paper, however, I shall restrict myself to integrals which are summable $\left(C_{r}\right)$, where $r$ is zero or a positive integer only. I propose to discuss the behaviour of $f(x)$ as $x \rightarrow \infty$, in such integrals. The main result is an extension (with a certain redundant condition removed) of a theorem by Dr. C. N. Moore in his paper on convergence factors. $\ddagger$

\section{§3. The Limit of the Integrand of a Summable Integral.}

In the case of an infinite series, it is a necessary but insufficient condition for convergence that $\lim _{n \rightarrow \infty} a_{n}=0$. More generally, for a series $\sum a_{n}$ to be summable $\left(C_{r}\right)$, it is necessary (though insufficient) that $\lim _{n \rightarrow \infty}\left(a_{n} / n^{r}\right)=0$; this has long been known to hold when $r$ is a positive integer, $\S$ and I have proved that it holds generally for any value of $r>-1$.

We should not expect the same result to hold in the case

* Comptes Rendus, July, 1909. Dr. Riesz's definition of the sum of a non-convergent series is

$$
\lim _{n \rightarrow \infty} \sum_{v=0}^{n-1} u_{v}\left(1-\frac{v}{n}\right)^{r}
$$

and evidently $n$ may be integral or not.

$\dagger$ "On non-integral orders . . . , "Proc. Lond. Math. Soc., 1911.

$\$$ The method also is an extension of the one there used, but the question itself was presented to me otherwise, by the corresponding theorem for series.

\& See, for instance, Bromwich, The Theory of Infinite Series, Ch. XI. 
of integrals, ${ }^{*}$ for even in the case of convergent integrals (when $r=0$ ) we know that $f(x)$ need not approach 0 , and in fact may have no finite upper limit. An example of this will be given almost immediately.

It is well-known, however, that if $\int_{0}^{\infty} f(x) d x$ converges, then the condition of uniform continuity of $f(x)$, for $x \geqq k$, where $k$ is any constant, is sufficient to ensure that $f(x) \rightarrow 0$ as $x \rightarrow \infty$.

Simple continuity of itself implies uniform continuity over any finite closed range of the variable, but this is evidently not the case over an infinite range.

Dr. Moore, who considered only integrals summable $\left(C_{1}\right)$, proved that if $f(x)$ is uniformly continuous for $x \geqq k>0$, and if $\int_{0}^{\infty} f(x) d x$ is summable $\left(C_{1}\right)$, then

$$
\lim _{x \rightarrow \infty} f(x) / x=0 \text {. }
$$

This is a particular case of the more general theorem which I proceed to establish; but first it must be mentioned that the restriction upon $f(x)$ of uniform continuity is unnecessarily stringent. It is sufficient that $f(x) / x$ should be uniformly continuous for $x \geqq k>0$. As Dr. Moore himself proved in the memoir referred to, if $f(x)$ is uniformly continuous for $x \geqq k>0$, then so also is $f(x) / x^{r}$, for any $r>0$; but the converse does not hold. 0 for

For example, consider the function $f(x)$ defined thus: $f(x)=$

$$
n+1 / n^{2} \leqq x \leqq(n+1)-1 /(n+1)^{2} ;
$$

${ }^{*}$ It may be interesting to point out that the method analogous to that which is successful in the case of series here fails completely. In proving the theorem for series, we use a forrnula for $a_{n}$ in terms of $S_{n}^{r}$, viz.,

$$
a=S_{n}^{(r)}-(r+1) S_{n-1}^{(r)}+\frac{(r+1) r}{1.2} S_{n-2}^{(r)}-\cdots
$$

to $r$ or $n+1$ terms according as $r$ is or is not a positive integer.

The integral analogue of this expression is easily seen to be

which is equal to

$$
\int_{0}^{x}(x-z)^{-(r+)} \int_{0}^{z} f(t)(z-t)^{r} d t d z
$$

$$
\int_{0}^{x} f(t) d t \int_{t}^{x}(z-t)^{r}(x-z)^{-(r+2)} d z=\int_{0}^{x} \frac{f(t)}{x-t} d t \int_{0}^{1} \theta r(1-\theta)^{-(r+2)} d \theta .
$$

The second integral converges for no value of $r$, and therefore the method is useless. 
and $f\left[n \pm(1-\theta) / n^{2}\right]=\theta \sqrt{ } n$ for $0<\theta<1$. $\int_{0}^{\infty} f(x) d x$ is convergent but $f(n)=\sqrt{ } n$, so that $f(x)$ does not $\rightarrow 0$ as $x \rightarrow \infty$, nor has it even a finite upper limit. $f(x)$ is continuous but not uniformly so, for $x \geqq k>0$. But since $\int_{0}^{\infty} f(x) d x$ is summable $\left(C_{0}\right)$, by the general condition of consistency it is summable $\left(C_{1}\right)$; also $f(x) / x \rightarrow 0$, which however is not deducible from Dr. Moore's theorem, since $f(x)$ is not uniformly continuous. On the other hand, $f(x) / x$ is uniformly continuous, and hence $f(x) / x \rightarrow 0$ does follow from the theorem as we shall prove it, with the unnecessary restriction removed.

\section{$\S 4$.}

Theorem. If $f(x) / x^{r}$ is uniformly continuous for $x \geqq k>0$, and $\int_{0}^{\infty} f(x) d x$ is summable $\left(C_{r}\right)$, then

$$
\lim _{x \rightarrow \infty} f(x) / x^{r}=0 .
$$

I restrict myself to the case when $r$ is a positive integer. There seems little doubt, however, that the theorem holds for all values of $n>-1$.

Let

$$
L_{r}(x)=\int_{a}^{x} f(\alpha)\left(1-\frac{\alpha}{x}\right)^{r} d \alpha .
$$

By hypothesis, if $L$ is the "sum" of the integral,

$$
L_{r}(x) \rightarrow L \text { as } x \rightarrow \infty .
$$

Then, $r$ being integral, we have

$$
f(x)=\frac{1}{r !} \frac{d^{r+1}}{d x^{r+1}}\left[x^{r} L_{r}(x)\right],
$$

which is immediately deducible from either form of $L_{r}(x)$. Since $f(x) / x^{r}$ is uniformly continuous for $x \geqq k>0$, we can find a positive number $\delta$, when any arbitrarily small positive constant $\epsilon$ is assigned, so that for $x \geqq k$,

$$
\left|\frac{f(x+h)}{(x+h)^{r}}-\frac{f(x)}{x^{r}}\right|<\epsilon,
$$

provided $0<|h|<(r+1) \delta$. 
But by the general mean value theorem of the differential calculus and equation (1) we have

$$
\text { (3) } \begin{aligned}
\sum_{m=0}^{r+1}(-1)^{m} & \left(\begin{array}{c}
\mathrm{r}+1 \\
m
\end{array}\right)(x+\overline{r+1-m \delta})^{r} L_{r}(x+\overline{r+1-m \delta}) \\
= & \delta^{r+1} \frac{d^{r+1}}{d x^{r+1}}\left[(x+\theta \delta)^{r} L_{r}(x+\theta \delta)\right] \\
= & r ! \delta^{r+1} f(x+\theta \delta)_{x} \quad(0<\theta<r+1) .
\end{aligned}
$$

We now expand the expression (3) in the form of an integral polynomial in $x$. We get

$$
\begin{gathered}
x^{r} \sum_{m=0}^{r}(-1)^{m}\left(\begin{array}{c}
r \\
m
\end{array}\right)\left\{L_{r}(x+\overline{r+1-m \delta})-L_{r}(x+\overline{r-m \delta})\right\} \\
+x^{r-1} S,
\end{gathered}
$$

where $S$ contains only a finite number of terms (a number fixed when $r$ is fixed) and, since $L_{r}(x) \rightarrow L$, has a finite upper limit $K$ for all values of $x$ and for all values of $\delta<1$, say. Further, we can find a constant $x_{0}$, depending on $\epsilon$ and $\delta$, such that for $x^{\prime}>x \geqq x_{0}$,

$$
\left|L_{r}\left(x^{\prime}\right)-L_{r}(x)\right|<\epsilon \delta^{r+1} .
$$

Hence for $x \geqq x_{0}$, we have

or

$$
r ! \delta^{r+1} f(x+\theta \delta)<2^{r} \cdot x^{r} \cdot \epsilon \cdot \delta^{r+1}+x^{r-1} S,
$$

$$
\frac{f(x+\theta \delta)}{x^{r}}<\frac{2^{r}}{r !} \epsilon+\frac{K}{. r ! \delta^{r+1} x} .
$$

Hence if $x_{0}>1 / \delta^{r+1} \epsilon$,

$$
\frac{f(x+\theta \delta)}{(x+\theta \delta)^{r}}<K^{\prime} \epsilon,
$$

where $K^{\prime}$ is independent of $x$ and $\delta$. Now $\theta$ is a function of $x$, and hence $x+\theta \delta$ does not necessarily assume all values along the real axis. But by equation (2) it follows that for all values of $x \geqq x_{0}>1 / \delta^{r+1} \epsilon$

$$
\frac{f(x)}{x^{r}}<K^{\prime \prime} \epsilon,
$$


where $K^{\prime \prime}$ is a positive constant, and consequently $f(x) / x^{r} \rightarrow 0$ as $x \rightarrow \infty$, which was what we desired to prove.

In conclusion, we may remark that the theorem may be stated as one of pure integral calculus, without reference to the theory of summability of integrals. Putting $f(x)=\phi(x) x^{r}$, the theorem thus becomes:

If $\phi(x)$ is uniformly continuous over the infinite interval $x \geqq k$ $>0$, then the convergence to a limit, as $x \rightarrow \infty$, of the integral

$$
\int_{0}^{x} \phi(\beta) \beta^{r}\left(1-\frac{\beta}{x}\right)^{r} d \beta
$$

requires that $\phi(x)$ shall $\rightarrow 0$ as $x \rightarrow \infty$.

CAMBRIDGE,

ENGLAND.

\section{IRREDUCIBLE HOMOGENEOUS LINEAR GROUPS OF ORDER $p^{m}$ AND DEGREE $p$ OR $p^{2}$.}

BY PROFESSOR W. B. FITE.

(Read before the American Mathematical Society, February 25, 1911.)

No group all of whose non-invariant commutators give invariant commutators besides identity can be simply isomorphic with irreducible groups of different degrees. This category includes all groups of order $p^{m}$ ( $p$ a prime) and classes one, two, and three. Moreover no group of order $p^{m}$ can be simply isomorphic with irreducible groups of just two different degrees.*

A consideration of these facts gives rise to the query as to whether any group of order $p^{m}$ can be simply isomorphic with irreducible groups of different degrees, and it is the purpose of this note to answer this question for certain special cases.

In the first place, if $G$ is an irreducible group of order $p^{m}$ and degree $p$, it cannot be simply isomorphic with an irreducible group of any other degree, since it contains an abelian subgroup of index $\uparrow p$, and since a group of order $p^{m}$ with an abelian sub-

* Bulletin, vol. 14 (1908), pp. 328, 329.

$\dagger$ Transactions Amer. Math. Society, vol. 7 (1906), p. 68. We shall have occasion to make use of the fact, established here, that in an irreducible group of order $p^{m}$ and degree $p$, the substitutions commutative with a substitution that gives an invariant commutator besides identity form an abelian subgroup. 